\title{
Biological Agent Strain Name
}

National Cancer Institute

\section{Source}

National Cancer Institute. Biological Agent Strain Name. NCI Thesaurus. Code C158305.

The strain name of the biological challenge agent. 